\title{
DISCLAIMER
}

This document was prepared as an account of work sponsored by the United States Government. While this document is believed to contain correct information, neither the United States Government nor any agency thereof, nor the Regents of the University of California, nor any of their employees, makes any warranty, express or implied, or assumes any legal responsibility for the accuracy, completeness, or usefulness of any information, apparatus, product, or process disclosed, or represents that its use would not infringe privately owned rights. Reference herein to any specific commercial product, process, or service by its trade name, trademark, manufacturer, or otherwise, does not necessarily constitute or imply its endorsement, recommendation, or favoring by the United States Government or any agency thereof, or the Regents of the University of California. The views and opinions of authors expressed herein do not necessarily state or reflect those of the United States Government or any agency thereof or the Regents of the University of California.

\section{Group velocity and pulse lengthening of mismatched laser pulses in plasma channels}

\author{
C. B. Schroeder, C. Benedetti, E. Esarey, J. van Tilborg, and W. P. Leemans \\ Lawrence Berkeley National Laboratory, Berkeley, California 94720, \\ USA
}

(Dated: 7 July 2011)

Analytic solutions are presented to the non-paraxial wave equation describing an ultra-short, low-power, laser pulse propagating in a plasma channel. Expressions for the laser pulse centroid motion and laser group velocity are derived, valid for matched and mismatched propagation in a parabolic plasma channel, as well as in vacuum, for an arbitrary Laguerre-Gaussian laser mode. The group velocity of a mismatched laser pulse, for which the laser spot size is strongly oscillating, is found to be independent of propagation distance and significantly less than that of a matched pulse. Laser pulse lengthening of a mismatched pulse owing to laser mode slippage is examined and found to dominate over that due to dispersive pulse spreading for sufficiently long pulses. Analytic results are shown to be in excellent agreement with numerical solutions of the full Maxwell equations coupled to the plasma response. Implications for plasma channel diagnostics are discussed.

PACS numbers: 52.38.-r, 52.38.Hb, 52.38.Kd 


\section{INTRODUCTION}

The propagation of tightly focused, short-pulse lasers in plasma is of fundamental importance. Plasma channels may be employed to optically guide short-pulse lasers, thereby extending the laser-plasma interaction length. ${ }^{1,2}$ Plasma channels consist of a plasma column with a minimum plasma density (i.e., an index of refraction that peaks) along the laser propagation axis. Extending the laser-plasma interaction length by plasma channel guiding is beneficial to a variety of applications, such as laser-plasma accelerators, ${ }^{3}$ direct laser acceleration, ${ }^{4,5}$ harmonic generation,,${ }^{6,7}$ and x-ray lasers. ${ }^{8-10}$ Critical to many of these applications is detailed knowledge of the group velocity of the laser propagating in the plasma channel. Plasma channels have experimentally demonstrated optical guiding over many Rayleigh lengths, and methods for plasma channel generation are actively being investigated. ${ }^{11-19}$

Previously it was shown ${ }^{20}$ that the linear group velocity $v_{g}=c \beta_{g}$ of a matched laser pulse propagating in a parabolic plasma channel is $v_{g}=v_{\text {match }}=c \beta_{\text {match }}$, where $\beta_{\text {match }}=$ $1-\left(k_{p} / k_{0}\right)^{2} / 2-2 /\left(k_{0} r_{0}\right)^{2}$, with $\omega_{0} \simeq c k_{0}=2 \pi c / \lambda_{0}$ the laser frequency, $\omega_{p}=c k_{p}=2 \pi c / \lambda_{p}=$ $\left(4 \pi n_{0} e^{2} / m_{e}\right)^{1 / 2}$ the plasma frequency, $n_{0}$ the on-axis plasma density, $e$ is the electron charge, $m_{e}$ is the electron mass, $c$ is the speed of light in vacuum, $r_{0}$ the matched laser spot size [for a Gaussian transverse profile, i.e., laser intensity $\left.\propto \exp \left(-2 r^{2} / r_{0}^{2}\right)\right]$, and $\left(k_{p} / k_{0}\right)^{2} \ll 1$ and $1 /\left(k_{0} r_{0}\right)^{2} \ll 1$ are assumed. Here, a parabolic plasma channel was assumed with a density profile of the form $n(r)=n_{0}+\Delta n r^{2} / r_{0}^{2}$, where $\Delta n$ is the channel depth. Propagation of a low intensity laser pulse at a constant laser spot size $r_{s}=r_{0}$ (i.e., matched propagation) is possible when $\Delta n=\Delta n_{c}$, where $\Delta n_{c}=\left(\pi r_{e} r_{0}^{2}\right)^{-1}$ is the critical depth and $r_{e}=e^{2} / m_{e} c^{2}$ is the classical electron radius. Matched propagation will only occur for a careful and exact choice (i.e., matching) of the laser spot size and divergence at the entrance of the plasma channel. If this matching is not achieved, mismatched laser propagation will result and the spot size $r_{s}$ oscillates as the laser propagates within the plasma channel. An oscillation in the laser spot size alters the laser group velocity.

The dependence of the laser group velocity on the plasma channel properties may serve as the basis of a plasma channel diagnostic. Recently a plasma channel diagnostic was proposed that relied on measuring the channel-induced delay between two laser pulses of two colors. ${ }^{21}$ By measuring the delay as a function of initial laser parameters, the on-axis plasma density 
and the channel depth (and, hence, the matched spot size) can be determined. For proper interpretation of this diagnostic technique, an accurate and general expression for the laser group velocity of a mismatched laser pulse is essential.

In this work a general expression for the linear group velocity of a mismatched laser pulse propagating in a parabolic plasma channel is derived in the low-power limit. It is shown that the group velocity of a mismatched laser pulse is significantly less than that of a matched pulse and is constant, i.e., independent of propagation distance, even though the laser spot size exhibits strong oscillations. Expressions valid for arbitrary Laguerre-Gaussian modes are derived. These analytical solutions are shown to be in good agreement to solutions of the full Maxwell-plasma equations. Nonlinear effects, such as those arising from laser evolution (e.g., depletion and red-shifting), are discussed. The nonlinear group velocity of a highintensity laser pulse in the one-dimensional limit, including the nonlinear plasma response, has been analyzed in Refs. 22 and 23.

This paper is organized as follows. In Sec. II a non-paraxial solution is presented for the wave equation of a laser propagating in a plasma channel. The lowest order non-paraxial operator is retained to describe group velocity effects (not present in the paraxial wave equation). In Sec. III a general expression for the linear laser group velocity is derived. The laser group velocity is defined as the velocity of the laser (normalized-intensity-weighted) centroid. It is shown that the laser group velocity is constant along the propagation. One consequence of the mode-dependent group velocity is mode dispersion of a mismatched laser pulse. Section IV examines the pulse lengthening via laser mode dispersion of a mismatched laser pulse. It is shown that this is the dominant lengthening mechanism for a mismatched laser pulse propagating in a plasma channel. Conclusions are presented in Sec. V. Also included are two Appendices. Appendix A discusses the limit of the absence of a plasma channel (i.e., a diffracting laser pulse in a uniform plasma), and Appendix B discusses the local laser velocity, which varies along the laser propagation direction and the radial distance from the axis of propagation. 


\section{NON-PARAXIAL LASER ENVELOPE EVOLUTION}

The wave equation describing the three-dimensional evolution of a laser pulse in a fullyionized plasma is ${ }^{20}$

$$
\left(\nabla^{2}-\partial_{c t}^{2}\right) \boldsymbol{a}_{\perp}=k_{p}^{2} \rho \boldsymbol{a}_{\perp}
$$

where $\boldsymbol{a}_{\perp}=e \boldsymbol{A}_{\perp} / m c^{2}$ is the normalized laser transverse vector potential with the gauge $\nabla \cdot \boldsymbol{a}=0$. The peak amplitude of the normalized laser vector potential is related to the laser intensity by $a_{0}^{2} \simeq 7.32 \times 10^{-19} \lambda_{0}^{2}[\mu \mathrm{m}] I_{0}\left[\mathrm{~W} / \mathrm{cm}^{2}\right]$ for a linearly polarized laser with $I_{0}$ the peak intensity. In general, the normalized proper density $\rho$ is a nonlinear function of the laser vector potential. ${ }^{3,23}$ In this paper we will consider the linear response of the plasma to the laser field, valid for $a^{2} \ll 1$, where $\rho \simeq n(r) / n_{0}$, i.e., the ambient plasma density normalized to the on-axis density. We will also consider the lower power limit $P / P_{c}=\left(a_{0} k_{p} r_{0}\right)^{2} / 32 \ll 1$, where $P_{c}$ is the critical power for relativistic self-focusing. ${ }^{3}$

A parabolic plasma density channel may be used to optically guide a Gaussian laser pulse. We consider propagation of the laser in a parabolic density channel of the form

$$
n(r)=n_{0}+\Delta n\left(r / r_{0}\right)^{2}=n_{0}+\left(\pi r_{e} r_{m}^{2}\right)^{-1}\left(r / r_{m}\right)^{2},
$$

with the matched radius related to the channel depth by $r_{m}=r_{0}\left(\Delta n_{c} / \Delta n\right)^{1 / 4}$. Such a plasma profile can guide a laser pulse of spot size $r_{0}$ provided the channel depth $\Delta n$ satisfies $\Delta n=\Delta n_{c}=\left(\pi r_{e} r_{0}^{2}\right)^{-1}$. In the following we will characterize the channel depth with the matched radius $r_{m}$, i.e., for $\Delta n=\Delta n_{c}$ and $r_{m}=r_{0}$. Longitudinal variation within the plasma channel is neglected. Note that a longitudinal variation in the on-axis density $n_{0}(z)$ can be included in a straightforward manner since the matched guiding condition is determined by only the channel depth. Effects of laser in-coupling and out-coupling are also neglected, which will be small provided that the coupling occurs over a scale length much less than the Rayleigh range.

Performing a variable transform to the co-moving variables, $(z, t) \rightarrow\left(\zeta=z-\beta_{g 0} c t, z\right)$, the wave equation Eq. (1) becomes

$$
\left[\nabla_{\perp}^{2}+2 \partial_{\zeta z}^{2}+\left(1-\beta_{g 0}^{2}\right) \partial_{\zeta}^{2}+\partial_{z}^{2}\right] \boldsymbol{a}_{\perp}=k_{p}^{2}(r) \boldsymbol{a}_{\perp}
$$

The laser vector potential may be decomposed into a slowly-varying envelope and fast phase, $\boldsymbol{a}_{\perp}=\hat{a} \exp \left(i k_{0} z-i \omega_{0} t\right) \hat{e}_{x}+$ c.c., such that the wave equation for the laser envelope is

$$
\left[\nabla_{\perp}^{2}+2\left(i k_{0}+\partial_{\zeta}\right) \partial_{z}+\left(1-\beta_{g 0}^{2}\right) \partial_{\zeta}^{2}+\partial_{z}^{2}\right] \hat{a}=\left[k_{p}^{2}(r)+\left(k_{0}^{2}-\omega_{0}^{2} / c^{2}\right)\right] \hat{a}
$$


and $\omega_{0} / k_{0} c=1 / \beta_{g 0}$ is assumed. The $\partial_{z}^{2} \hat{a}$ term may be neglected assuming a slowly-varying envelope such that $\left|\partial_{\zeta} \hat{a}\right| \gg\left|\partial_{z} \hat{a}\right|$, i.e., a short-pulse laser with $L \ll Z_{R}$, where $L$ is the laser pulse length and $Z_{R}=k_{0} r_{0}^{2} / 2$ is the Rayleigh range with $r_{0}$ the laser focal spot and $k_{0}$ the central wavenumber of the laser. In this work we will also neglect the term in the wave equation Eq. (4) responsible for dispersion, $\left(1-\beta_{g 0}^{2}\right) \partial_{\zeta}^{2} \hat{a}$. Keeping only the lowest-order non-paraxial correction $\partial_{\zeta z}^{2} \hat{a}$, the wave equation becomes

$$
\left[\nabla_{\perp}^{2}+2\left(i k_{0}+\partial_{\zeta}\right) \partial_{z}\right] \hat{a}=\left[k_{p}^{2}(r)-k_{0}^{2}\left(\beta_{g 0}^{-2}-1\right)\right] \hat{a}
$$

Taking a Fourier transform with respect to $\zeta$ yields

$$
\left[\nabla_{\perp}^{2}+2 i\left(k_{0}+\delta k\right) \partial_{z}\right] \hat{a}_{k}=\left[k_{p}^{2}(r)-k_{0}^{2}\left(\beta_{g 0}^{-2}-1\right)\right] \hat{a}_{k}
$$

where

$$
\hat{a}_{k}=\int_{-\infty}^{\infty} d \zeta e^{-i \zeta \delta k} \hat{a}(\zeta) .
$$

The transformed wave equation Eq. (6) has the general solution in cylindrical coordinates $(r, \phi, z)$ of a Laguerre-Gaussian mode, characterized by mode numbers $m$ and $p$,

$$
\hat{a}_{k}=b_{k}\left(2 r^{2} / r_{s}^{2}\right)^{p / 2} L_{m}^{p}\left(2 r^{2} / r_{s}^{2}\right) e^{\left[i \theta-(1-i \alpha) r^{2} / r_{s}^{2}+i p \phi\right]},
$$

where $L_{m}^{p}(x)=\left(e^{x} x^{-p} / m !\right) \partial_{x}^{(m)}\left(e^{-x} x^{m+p}\right)$ are the generalized Laguerre polynomials. Substituting Eq. (8) into Eq. (6) yields the equations,

$$
\begin{aligned}
\frac{\partial\left(b_{k} r_{s}\right)}{\partial z} & =0 \\
\alpha & =\frac{k r_{s}}{2} \frac{\partial r_{s}}{\partial z} \\
\frac{\partial \theta}{\partial z} & =-\frac{2}{k}(1+2 m+p)\left(\frac{1}{r_{s}^{2}}-\frac{1}{r_{m}^{2}}\right) \\
\frac{\partial^{2} r_{s}}{\partial z^{2}} & =\frac{4}{k^{2} r_{s}^{3}}\left(1-\frac{r_{s}^{4}}{r_{m}^{4}}\right)
\end{aligned}
$$

for the laser amplitude (in Fourier space) $b_{k}$, curvature $\alpha$, phase shift $\theta$, and laser spot size $r_{s}$, respectively. Here $k=k_{0}+\delta k$. Derivation of Eq. (11) assumed

$$
\beta_{g 0}^{2}=1-\frac{k_{p}^{2}}{k_{0}^{2}}-\frac{4(1+2 m+p)}{k_{0}^{2} r_{m}^{2}}
$$


For the initial conditions $\alpha(z=0)=0, r_{s}(z=0)=r_{i}, \partial_{z} r_{s}(z=0)=0$, and $\theta(z=0)=0$, the solutions to Eqs. (9)-(12) are $b_{k}=b_{k 0} r_{i} / r_{s}$,

$$
\begin{aligned}
\alpha(k) & =-\frac{1}{2}\left(\frac{r_{i}^{2}}{r_{m}^{2}}-\frac{r_{m}^{2}}{r_{i}^{2}}\right) \sin \left(2 z / Z_{R M}\right), \\
\theta(k) & =(1+2 m+p)\left\{\frac{z}{Z_{R M}}-\tan ^{-1}\left[\frac{r_{m}^{2}}{r_{i}^{2}} \tan \left(\frac{z}{Z_{R M}}\right)\right]\right\}, \\
r_{s}^{2}(k) & =\frac{r_{i}^{2}}{2}\left[\left(1+\frac{r_{m}^{4}}{r_{i}^{4}}\right)+\left(1-\frac{r_{m}^{4}}{r_{i}^{4}}\right) \cos \left(2 z / Z_{R M}\right)\right],
\end{aligned}
$$

with $Z_{R M}=k r_{m}^{2} / 2$ and $b_{k 0}$ is the initial spectrum of the laser at $z=0$. The above initial conditions correspond to the laser entering the plasma channel at an extrema of the laser spot size oscillation. As we will show in Sec. III these initial conditions may be taken without loss of generality by using the first integral of Eq. (12).

The solution for the laser envelope is given by the inverse Fourier transform of $\hat{a}_{k}$,

$$
\hat{a}=\int_{-\infty}^{\infty} \frac{d \delta k}{2 \pi} e^{i \zeta \delta k} \hat{a}_{k}=\int_{-\infty}^{\infty} \frac{d \delta k}{2 \pi} e^{i \zeta \delta k} b_{k 0} e^{\psi},
$$

with the exponent

$$
\psi(k)=\ln \left[\frac{r_{i}}{r_{s}} \chi^{p / 2} L_{m}^{p}(\chi)\right]+i \theta-(1-i \alpha) \chi / 2+i p \phi
$$

and $\chi=2 r^{2} / r_{s}^{2}$. Consider an expansion of the exponent $\psi$ about the paraxial solution

$$
\psi(k) \simeq \psi\left(k_{0}\right)+\psi^{\prime}\left(k_{0}\right) \delta k,
$$

with $\psi^{\prime}=\partial_{k} \psi$, such that the lowest order non-paraxial term is retained. Then the laser envelope solution Eq. (17) becomes

$$
\hat{a}=\int_{\infty}^{\infty} \frac{d \delta k}{2 \pi} e^{i \zeta \delta k} b_{k 0} e^{\psi\left(k_{0}\right)+\psi^{\prime}\left(k_{0}\right) \delta k}=b_{0}\left(\zeta-i \psi^{\prime}\right) e^{\psi\left(k_{0}\right)} .
$$

Assuming an initial Gaussian pulse profile, $b_{0}(\zeta)=a_{0} \exp \left(-\zeta^{2} / L^{2}\right)$, the intensity profile for the non-paraxial solution is

$$
|\hat{a}|^{2}=a_{0}^{2} e^{2 \Re\left[\psi\left(k_{0}\right)\right]} e^{-2\left(\zeta+\Im\left[\psi^{\prime}\left(k_{0}\right)\right)^{2} / L^{2}\right.} e^{-2 \Re\left[\psi^{\prime}\left(k_{0}\right)\right]^{2} / L^{2}},
$$

where $\Re$ and $\Im$ denote the real and imaginary components, respectively. The term $\left|\Re\left[\psi^{\prime}\left(k_{0}\right)\right]^{2} / L^{2}\right| \sim 1 /\left(k_{0} L\right)^{2} \ll 1$, which describes pulse length effects, may be neglected provided $k_{0} L \gg 1$. The paraxial exponent is

$$
\psi\left(k_{0}\right)=\ln \left[\frac{r_{i}}{r_{s 0}} \chi_{0}^{p / 2} L_{m}^{p}\left(\chi_{0}\right)\right]+i \theta_{0}-\left(1-i \alpha_{0}\right) \frac{\chi_{0}}{2}+i p \phi,
$$




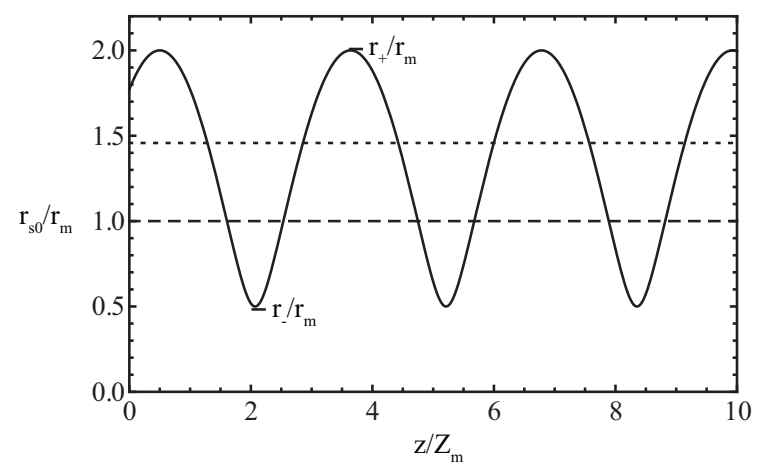

FIG. 1. Laser spot size evolution in a plasma channel, $r_{s 0} / r_{m}$ versus $z / Z_{m}$, for a mismatched laser (solid curve), with spot size extrema $r_{+} / r_{m}=2$ and $r_{-} / r_{m}=1 / 2$, and for a matched laser pulse $r_{i}=r_{m}$ (dashed line). The rms radius of the mismatched pulse $\left\langle r_{s 0}^{2}\right\rangle^{1 / 2} / r_{m}=\left\{\left[\left(r_{ \pm} / r_{m}\right)^{2}+\right.\right.$ $\left.\left.\left(r_{m} / r_{ \pm}\right)^{2}\right] / 2\right\}^{1 / 2}$ (dotted line) is also shown.

where $\chi_{0}=2 r^{2} / r_{s 0}^{2}, \alpha_{0}=\alpha\left(k_{0}\right), \theta_{0}=\theta\left(k_{0}\right)$, and $r_{s 0}=r_{s}\left(k_{0}\right)$. The imaginary component of the non-paraxial exponent is

$$
\Im\left[\psi^{\prime}\left(k_{0}\right)\right]=k_{0}^{-1}\left(\Theta_{1}+\Theta_{2} \chi_{0}\right),
$$

where

$$
\begin{aligned}
\Theta_{1} & =-(1+2 m+p) \frac{z}{Z_{m}}\left[1-\frac{r_{i}^{2} r_{m}^{2} \sec ^{2}\left(z / Z_{m}\right)}{r_{i}^{4}+r_{m}^{4} \tan ^{2}\left(z / Z_{m}\right)}\right] \\
\Theta_{2} & =\frac{\left(z / Z_{m}\right)\left[\left(r_{m}^{4}-r_{i}^{4}\right)^{2}-\left(r_{m}^{8}-r_{i}^{8}\right) \cos \left(2 z / Z_{m}\right)\right]}{2 r_{i}^{2} r_{m}^{2}\left[r_{m}^{4}+r_{i}^{4}-\left(r_{m}^{4}-r_{i}^{4}\right) \cos \left(2 z / Z_{m}\right)\right]},
\end{aligned}
$$

with $Z_{m}=k_{0} r_{m}^{2} / 2$ the Rayleigh range of the matched radius. Using $\Re\left[\psi\left(k_{0}\right)\right]$, the solution to the non-paraxial laser envelope is

$$
|\hat{a}|^{2}=a_{0}^{2} \frac{r_{i}^{2}}{r_{s 0}^{2}} \chi_{0}^{p}\left[L_{m}^{p}\left(\chi_{0}\right)\right]^{2} e^{-\chi_{0}} \exp \left[-2\left(\zeta+\Im\left[\psi^{\prime}\right]\right)^{2} / L^{2}\right],
$$

with the imaginary component of the non-paraxial exponent given by Eqs. (23)-(25). The general solution in the limit $\Delta n \rightarrow 0$ (or $r_{m} \rightarrow \infty$ ), with $r_{i}=r_{0}$, i.e., a diffracting laser pulse without a plasma channel, is presented in Appendix A.

For a mismatched laser in a plasma channel the laser spot size oscillates as it propagates in the channel. Figure 1 shows the laser spot size $r_{s 0} / r_{m}$ evolution in a plasma channel for a mismatched laser (with spot size extrema $r_{+} / r_{m}=2$ and $r_{-} / r_{m}=1 / 2$ ). The dashed line in Fig. 1 is the radius of a matched laser pulse $r_{i}=r_{m}$, and the dotted line is the rms 
radius of the mismatched pulse $\left\langle r_{s 0}^{2}\right\rangle^{1 / 2} / r_{m}=\left\{\left[\left(r_{ \pm} / r_{m}\right)^{2}+\left(r_{m} / r_{ \pm}\right)^{2}\right] / 2\right\}^{1 / 2}$. In the next section the non-paraxial solution for the laser intensity Eq. (26) is used to calculate the laser group velocity, and it is shown that the larger effective transverse wavenumber owing to the laser spot oscillations $\left\langle r_{s 0}^{2}\right\rangle^{1 / 2}>r_{m}$ results in a slower laser group velocity for fixed channel parameters.

\section{LASER GROUP VELOCITY}

Here the laser group velocity is defined as the transport velocity of the global centroid

of the laser. The global laser centroid, in the co-moving variable, weighted by the square of the laser vector potential, is

$$
\langle\zeta\rangle=\frac{\int_{0}^{\infty} r d r \int_{-\infty}^{\infty} d \zeta|\hat{a}|^{2} \zeta}{\int_{0}^{\infty} r d r \int_{-\infty}^{\infty} d \zeta|\hat{a}|^{2}}
$$

Note that a local laser centroid may also be defined, as discussed in Appendix B. Using Eq. (26), the laser centroid is

$$
\begin{aligned}
\langle\zeta\rangle & =\frac{\int_{0}^{\infty} d \chi_{0}\left(-\Im\left[\psi^{\prime}\right]\right) e^{-\chi_{0}} \chi_{0}^{p}\left[L_{m}^{p}\left(\chi_{0}\right)\right]^{2}}{\int_{0}^{\infty} d \chi_{0} e^{-\chi_{0}} \chi_{0}^{p}\left[L_{m}^{p}\left(\chi_{0}\right)\right]^{2}} \\
& =-k_{0}^{-1}\left[\Theta_{1}+\Theta_{2}(1+2 m+p)\right] .
\end{aligned}
$$

Evaluating Eq. (28) with Eqs. (24) and (25) yields

$$
k_{0}\langle\zeta\rangle=-\frac{z}{Z_{m}} \frac{\left(r_{i}^{2}-r_{m}^{2}\right)^{2}}{2 r_{m}^{2} r_{i}^{2}}(1+2 m+p) .
$$

The group velocity associated with the rate of change of the laser centroid, with respect to $\beta_{g 0}$, is

$$
\begin{aligned}
\beta_{G} & =\beta_{g 0}+\frac{d\langle\zeta\rangle}{d z} \\
& =1-\frac{k_{p}^{2}}{2 k_{0}^{2}}-\frac{(1+2 m+p)}{k_{0}^{2} r_{i}^{2}}\left(1+\frac{r_{i}^{4}}{r_{m}^{4}}\right) .
\end{aligned}
$$

Equation (30) can be generalized to include an initially converging or diverging laser by considering the first integral of the laser spot evolution equation Eq. (12),

$$
\frac{2\left\langle r_{s 0}^{2}\right\rangle}{r_{m}^{2}}=\frac{r_{m}^{2}}{r_{ \pm}^{2}}+\frac{r_{ \pm}^{2}}{r_{m}^{2}}=\frac{r_{m}^{2}}{r_{i}^{2}}+\frac{r_{i}^{2}}{r_{m}^{2}}+\frac{Z_{m}^{2}}{r_{m}^{2}}\left(\frac{\partial r_{i}}{\partial z}\right)^{2}
$$


where $\partial_{z} r_{i}=\partial_{z} r_{s}(z=0), r_{i}=r_{s}(z=0)$, and $r_{ \pm}$are the extrema of the spot size (where $\partial_{z} r_{s}=0$ ). Hence, the group velocity of a laser propagating in a plasma channel (generalized to include an initially converging or diverging laser at the channel entrance) is

$$
\beta_{G}=1-\frac{k_{p}^{2}}{2 k_{0}^{2}}\left[1+\frac{4(1+2 m+p)}{k_{p}^{2} r_{m}^{2}} \frac{\left\langle r_{s 0}^{2}\right\rangle}{r_{m}^{2}}\right] \text {. }
$$

Equation (32) is the main result of this work, and may be expressed in terms of the channel depth as

$$
\beta_{G}=1-\frac{k_{p}^{2}}{2 k_{0}^{2}}-\frac{(1+2 m+p)}{k_{0}^{2} r_{0}^{2}}\left[\frac{r_{0}^{2}}{r_{i}^{2}}\left(1+\frac{r_{i}^{4}}{r_{0}^{4}} \frac{\Delta n}{\Delta n_{c}}\right)+\frac{Z_{R}^{2}}{r_{0}^{2}}\left(\frac{\partial r_{i}}{\partial z}\right)^{2}\right]
$$

Note that the laser group velocity (global laser centroid velocity) is constant (independent of $z$ ) during the laser propagation. In addition, the velocity of the higher-order laser modes are slower than the fundamental Gaussian mode $(m=p=0)$ for the same initial conditions.

The general expression of the laser group velocity Eq. (32) [or Eq. (33)] reduces to previously derived results ${ }^{20}$ in the appropriate limits. In particular, for a matched laser in a channel, $\Delta n=\Delta n_{c}$ and the initial laser spot $r_{i}=r_{0}=r_{m}$ is the matched radius, the laser group velocity is

$$
\beta_{G}=1-\frac{k_{p}^{2}}{2 k_{0}^{2}}-\frac{2(1+2 m+p)}{k_{0}^{2} r_{m}^{2}} .
$$

For a diffracting laser (no plasma channel present), $\Delta n=0\left[\right.$ or $\left.r_{m}=r_{0}\left(\Delta n_{c} / \Delta n\right)^{1 / 4} \rightarrow \infty\right]$, $r_{i}=r_{0}$ is the minimum spot at focus, and the laser group velocity is

$$
\beta_{G}=1-\frac{k_{p}^{2}}{2 k_{0}^{2}}-\frac{(1+2 m+p)}{k_{0}^{2} r_{0}^{2}} .
$$

Figure 2 shows a comparison between the approximate analytical solution for the laser group velocity Eq. (32) (dashed lines) and a numerical solution (solid lines) to the full Maxwell equations coupled to the plasma response calculated using the code INF\&RNO. ${ }^{24}$ INF\&RNO is a 2D cylindrical code that solves the full wave equation for the laser envelope evolution and the cold plasma fluid-Maxwell equations for the plasma response in the $\left(k_{p} r, k_{p}(z-c t)\right)$ variables. The plasma responds to the laser field via the averaged laser ponderomotive force. ${ }^{25}$ Additional details on the numerics of INF\&RNO may be found in Ref. 24. Plotted in Fig. 2 is the quantity $\beta_{G}-1$ versus propagation distance $z / Z_{m}$ with $k_{0} / k_{p}=25, \lambda_{0}=0.8 \mu \mathrm{m}, k_{p} L=3.2$, and $a_{0}=0.01$, for the cases: (a) fundamental Gaussian laser mode diffracting in vacuum with $r_{0}=r_{m}$, (b) $m=1, p=0$ laser mode diffracting 


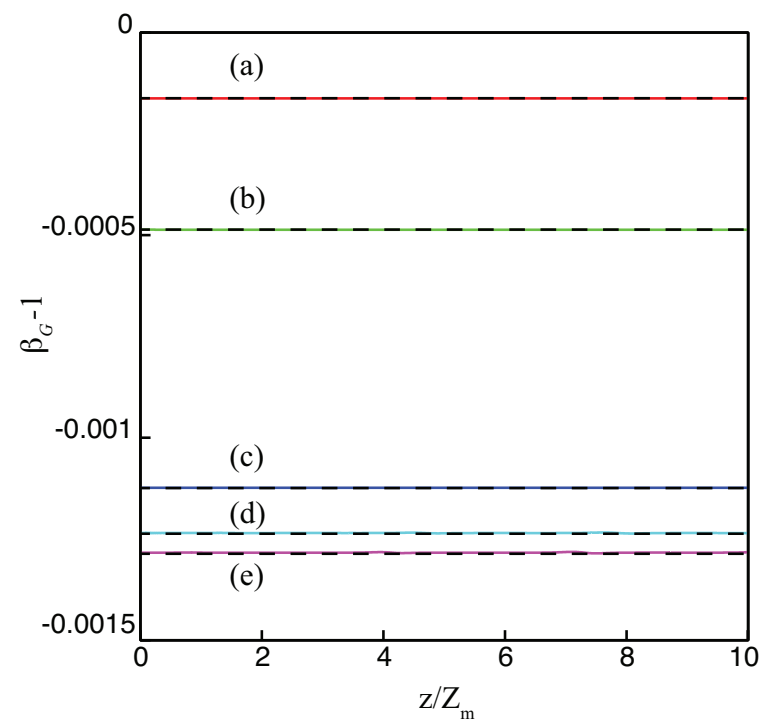

FIG. 2. The quantity $\beta_{G}-1$ versus propagation distance $z / Z_{m}$ with $k_{0} / k_{p}=25, \lambda_{0}=0.8 \mu \mathrm{m}$, $k_{p} L=3.2$, and $a_{0}=0.01$, for the cases: (a) vacuum diffraction of fundamental mode with $k_{p} r_{0}=\pi$ (here $Z_{m}=Z_{R}$ ), (b) $m=1, p=0$ laser mode diffracting in vacuum with $k_{p} r_{0}=\pi$, (c) matched laser in plasma channel with $k_{p} r_{i}=k_{p} r_{m}=\pi$, (d) mismatched laser in plasma channel with $k_{p} r_{i}=$ $1.5 k_{p} r_{m}=3 \pi / 2$, and (e) a converging laser into a plasma channel with $k_{p} r_{m}=\pi, r_{i}=\sqrt{2} r_{0}$, and $Z_{R} \partial_{z} r_{i}=-r_{0} / \sqrt{2}$. Dashed curves are analytic solutions Eq. (32), and solid curves are numerical solutions of the full Maxwell equations coupled to plasma response.

in vacuum with $r_{0}=r_{m}$, (c) matched laser in plasma channel with $k_{p} r_{i}=k_{p} r_{m}=\pi$, (d) mismatched laser in plasma channel with $r_{i}=1.5 r_{m}, k_{p} r_{m}=\pi$, and (e) a converging laser into a plasma channel with $k_{p} r_{m}=\pi, r_{i}=\sqrt{2} r_{0}$, and $Z_{R} \partial_{z} r_{i}=-r_{0} / \sqrt{2}$ (i.e., vacuum focus one Rayleigh range past the entrance of the plasma channel). Figure 2 shows excellent agreement between the numerical results and the analytic solution.

Although the laser group velocity Eq. (32) is valid in the linear regime $a_{0}^{2} \ll 1$, the group velocity in the weakly-relativistic regime $a_{0}^{2} \lesssim 1$ is reasonably well-approximated by the above theory over several Rayleigh ranges. This is illustrated in Fig. 3, which shows the analytic solutions Eq. (32) (dashed curves) and the numerical solutions of the full Maxwell equations coupled to plasma response (solid curves) for the cases of (i) matched and (ii) mismatched laser propagation in a plasma channel for initial laser intensities: $a_{0}=0.01$, $a_{0}=0.1, a_{0}=0.3$, and $a_{0}=0.5$. The deviation of the group velocity from the linear result is due to laser frequency shifts, i.e., redshifting as the laser deposits energy into the plasma via 


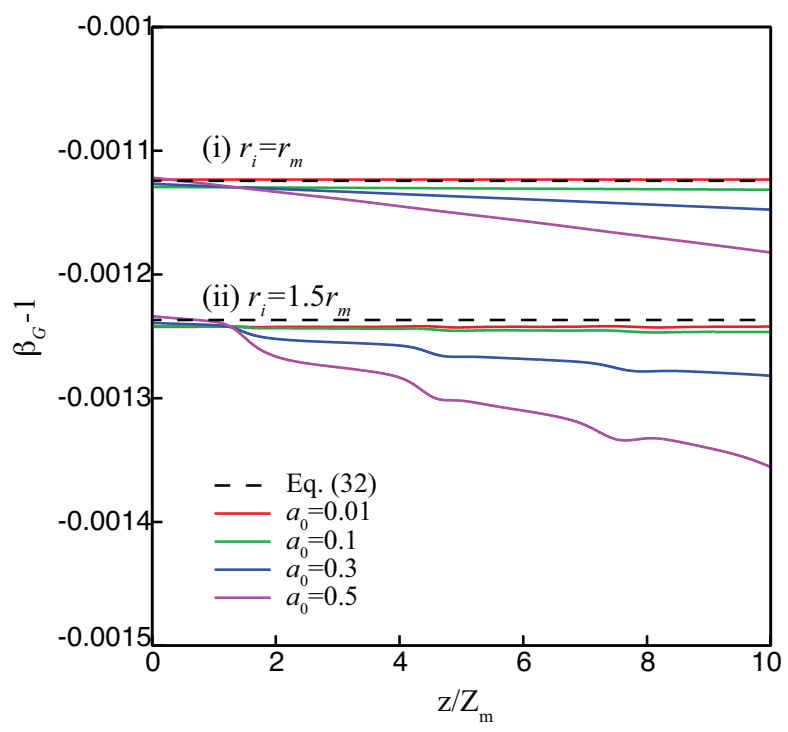

FIG. 3. The quantity $\beta_{G}-1$ versus propagation distance $z / Z_{m}$ with $k_{0} / k_{p}=25, \lambda_{0}=0.8 \mu \mathrm{m}$, and $k_{p} L=1$, for the cases: (i) matched laser in plasma channel with $k_{p} r_{i}=k_{p} r_{m}=\pi$ and (ii) mismatched laser in plasma channel with $k_{p} r_{i}=1.5 k_{p} r_{m}=3 \pi / 2$, for initial intensities $a_{0}=0.01$, $a_{0}=0.1, a_{0}=0.3$, and $a_{0}=0.5$. Dashed curves are analytic solutions Eq. (32), and solid curves are numerical solutions of the full Maxwell equations coupled to plasma response.

plasma wave excitation. ${ }^{23}$ Note that, for the parameters of Fig. 3, plasma wave excitation is significant since $k_{p} L=1$. Plasma wave effects on the laser, including red-shifting and laser energy depletion, can be reduced when $\left(P / P_{c}\right)\left(k_{p} L\right)^{2} \ll 1$.

\section{PULSE LENGTHENING VIA MODE DISPERSION}

As Eq. (34) indicates, the higher-order matched laser modes propagate at different group velocities. In general, a mismatched laser in a plasma channel may be decomposed into a series of higher-order matched laser modes. Therefore, a mismatched laser propagating in a plasma channel will lengthen as the higher-order modes slip with respect to each other. As is shown in this section, the scale length for the pulse lengthening of a mismatched laser is $\sim Z_{R} k_{0} L$. This scale length is much shorter than pulse lengthening owing to dispersion, ${ }^{20}$ which scales as $\sim Z_{R}\left(k_{0} L\right)^{2}$.

Consider the case of a laser propagating in a plasma channel and entering the channel at $z=0$ with mismatch $r_{i}=r_{m}+\delta r$. We will assume $\delta r \ll r_{m}$, and consider a fundamental 
Gaussian mode. Expanding Eq. (8) (with $m=p=0$ ), using Eqs. (14)-(16), yields $\hat{a}_{k}=$ $\hat{a}_{k 0}+\hat{a}_{k 1}\left(\delta r / r_{m}\right)+\hat{a}_{k 2}\left(\delta r / r_{m}\right)^{2}$, with

$$
\begin{aligned}
& \hat{a}_{k 0}=b_{k 0} e^{-\chi_{m} / 2}, \\
& \hat{a}_{k 1}=-b_{k 0} e^{-\chi_{m} / 2} L_{1}^{0}\left(\chi_{m}\right) e^{-i k_{\beta} z}, \\
& \hat{a}_{k 2}=-b_{k 0} e^{-\chi_{m} / 2}\left[1-L_{1}^{0}\left(\chi_{m}\right) e^{-i k_{\beta} z}-2 L_{2}^{0}\left(\chi_{m}\right) e^{-2 i k_{\beta} z}\right] / 2,
\end{aligned}
$$

where $k_{\beta}=2 / Z_{R M}$ and $\chi_{m}=2 r^{2} / r_{m}^{2}$. Retaining only the lowest-order non-paraxial terms, i.e., let $k_{\beta}=k_{\beta 0}\left(1-\delta k / k_{0}\right)$, and taking the inverse Fourier transform yields $\hat{a}=\hat{a}_{(0)}+$ $\hat{a}_{(1)}\left(\delta r / r_{m}\right)+\hat{a}_{(2)}\left(\delta r / r_{m}\right)^{2}$ with

$$
\begin{aligned}
& \hat{a}_{(0)}=b_{0}(\zeta) e^{-\chi_{m} / 2}, \\
& \hat{a}_{(1)}=-b_{0}\left(\zeta_{1}\right) e^{-\chi_{m} / 2} L_{1}^{0}\left(\chi_{m}\right) e^{-i k_{\beta 0} z}, \\
& \hat{a}_{(2)}=-e^{-\chi_{m} / 2}\left[b_{0}(\zeta)-b_{0}\left(\zeta_{1}\right) L_{1}^{0}\left(\chi_{m}\right) e^{-i k_{\beta 0} z}-2 b_{0}\left(\zeta_{2}\right) L_{2}^{0}\left(\chi_{m}\right) e^{-2 i k_{\beta 0} z}\right] / 2,
\end{aligned}
$$

where $k_{\beta 0}=2 / Z_{m}, \zeta_{1}=\zeta+z k_{\beta 0} / k_{0}$, and $\zeta_{2}=\zeta+2 z k_{\beta 0} / k_{0}$. Here the mismatched fundamental mode laser is composed of $m=0, m=1$, and $m=2$ (matched) Laguerre modes. The normalized laser pulse intensity is $|\hat{a}|^{2}=\left|a_{(0)}\right|^{2}+\left(a_{(0)}^{*} a_{(1)}+a_{(1)}^{*} a_{(0)}\right)\left(\delta r / r_{m}\right)+\left(a_{(0)}^{*} a_{(2)}+\right.$ $\left.a_{(2)}^{*} a_{(0)}+\left|a_{(1)}\right|^{2}\right)\left(\delta r / r_{m}\right)^{2}=I_{(0)}+I_{(1)}\left(\delta r / r_{m}\right)+I_{(2)}\left(\delta r / r_{m}\right)^{2}$, with

$$
\begin{aligned}
I_{(0)}= & b_{0}(\zeta)^{2} e^{-\chi_{m}}, \\
I_{(1)}= & -2 b_{0}\left(\zeta_{1}\right) b_{0}(\zeta) e^{-\chi_{m}} L_{1}^{0}\left(\chi_{m}\right) \cos \left(k_{\beta 0} z\right), \\
I_{(2)}= & -e^{-\chi_{m}}\left[b_{0}(\zeta)^{2}-b_{0}\left(\zeta_{1}\right)^{2}\left[L_{1}^{0}\left(\chi_{m}\right)\right]^{2}\right. \\
& -b_{0}\left(\zeta_{1}\right) b_{0}(\zeta) L_{1}^{0}\left(\chi_{m}\right) \cos \left(k_{\beta 0} z\right) \\
& \left.-2 b_{0}\left(\zeta_{2}\right) b_{0}(\zeta) L_{2}^{0}\left(\chi_{m}\right) \cos \left(2 k_{\beta 0} z\right)\right] .
\end{aligned}
$$

In the following we will consider an initial Gaussian laser pulse profile $b_{0}(\zeta)=a_{0} \exp \left(-\zeta^{2} / L^{2}\right)$.

The root-mean square (rms) laser pulse length is $\sigma=\left[\left\langle\zeta^{2}\right\rangle-\langle\zeta\rangle^{2}\right]^{1 / 2}$, where the intensityweighted centroid is, using Eqs. (42)-(44),

$$
\langle\zeta\rangle=\frac{\int_{0}^{\infty} r d r \int_{-\infty}^{\infty} d \zeta|\hat{a}|^{2} \zeta}{\int_{0}^{\infty} r d r \int_{-\infty}^{\infty} d \zeta|\hat{a}|^{2}}=-\frac{k_{\beta 0} z}{k_{0}}\left(\frac{\delta r}{r_{m}}\right)^{2} .
$$

Note that $k_{0}\langle\zeta\rangle=-k_{\beta 0} z\left(\delta r / r_{m}\right)^{2}=-2\left(z / Z_{m}\right)\left(\delta r / r_{m}\right)^{2}$ is in agreement with Eq. (29) in the limit of a slightly mismatched channel $r_{i} / r_{m}-1=\delta r / r_{m} \ll 1$. Evaluating the second 


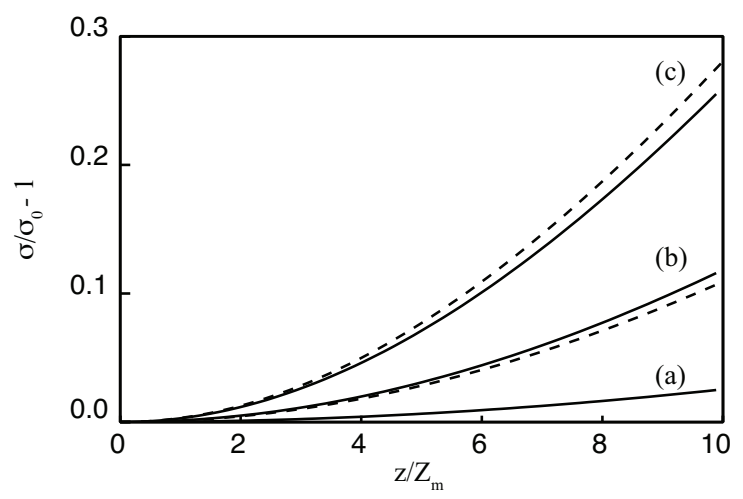

FIG. 4. Laser pulse rms length normalized to the initial value $\sigma / \sigma_{0}$ versus propagation distance $z / Z_{m}$ in a plasma channel for (a) a matched laser pulse $\left(r_{i}=r_{m}\right)$, (b) a mismatched laser pulse with $r_{i}=1.3 r_{m}$, and (c) mismatched laser pulse with $r_{i}=1.5 r_{m}$. Laser and plasma parameters are $k_{0} / k_{p}=25, k_{p} L=1, a_{0}=0.01$, and $\lambda_{0}=0.8 \mu \mathrm{m}$. Solid curves are numerical solutions of the full Maxwell equations coupled to the plasma response and dashed curves are calculated using the approximate solution Eq. (47).

moment of the co-moving variable $\left\langle\zeta^{2}\right\rangle$ using Eqs. (42)-(44) yields

$$
\left\langle\zeta^{2}\right\rangle=\frac{\int_{0}^{\infty} r d r \int_{-\infty}^{\infty} d \zeta|\hat{a}|^{2} \zeta^{2}}{\int_{0}^{\infty} r d r \int_{-\infty}^{\infty} d \zeta|\hat{a}|^{2}}=\frac{L^{2}}{4}+\left(\frac{k_{\beta 0} z}{k_{0}}\right)^{2}\left(\frac{\delta r}{r_{m}}\right)^{2}
$$

To order $\left(\delta r / r_{m}\right)^{2}$, the rms laser pulse length normalized to the initial value is

$$
\frac{\sigma}{\sigma_{0}}=\left[1+\left(\frac{4 z}{Z_{m} k_{0} L}\right)^{2}\left(\frac{\delta r}{r_{m}}\right)^{2}\right]^{1 / 2}
$$

where $\sigma_{0}=L / 2$ is the initial rms pulse length. Equation (47) may be generalized for a small mismatch owing to a converging or diverging laser into a plasma channel using the relation Eq. (31). Note that, for a matched pulse $r_{i}=r_{m}$, the rms pulse length is constant $\sigma=\sigma_{0}$. This is because we have neglected dispersion, i.e., the term $\left(1-\beta_{g 0}^{2}\right) \partial_{\zeta}^{2} \hat{a}$ in the wave equation Eq. (4), which appears as a non-paraxial term of order $\left(\delta k / k_{0}\right)^{2}$. The characteristic scale length for pulse lengthening via mode slippage is, from Eq. (47), $Z_{S}=\left(Z_{m} k_{0} L / 4\right)\left(r_{m} / \delta r\right)$. This scale length is much shorter than the scale length for the pulse to disperse via the laser bandwidth, ${ }^{20} Z_{D}=Z_{m}\left(k_{0} L\right)^{2} /\left(4+k_{p}^{2} r_{m}^{2}\right)$, provided the pulse length is sufficiently long, $k_{0} L \gg\left(1+k_{p}^{2} r_{m}^{2} / 4\right) r_{m} / \delta r$.

Figure 4 shows the rms laser pulse length normalized to the initial value $\sigma / \sigma_{0}$ versus propagation distance $\left(z / Z_{m}\right)$ in a plasma channel for (a) a matched laser pulse $\left(r_{i}=r_{m}\right)$, (b) 

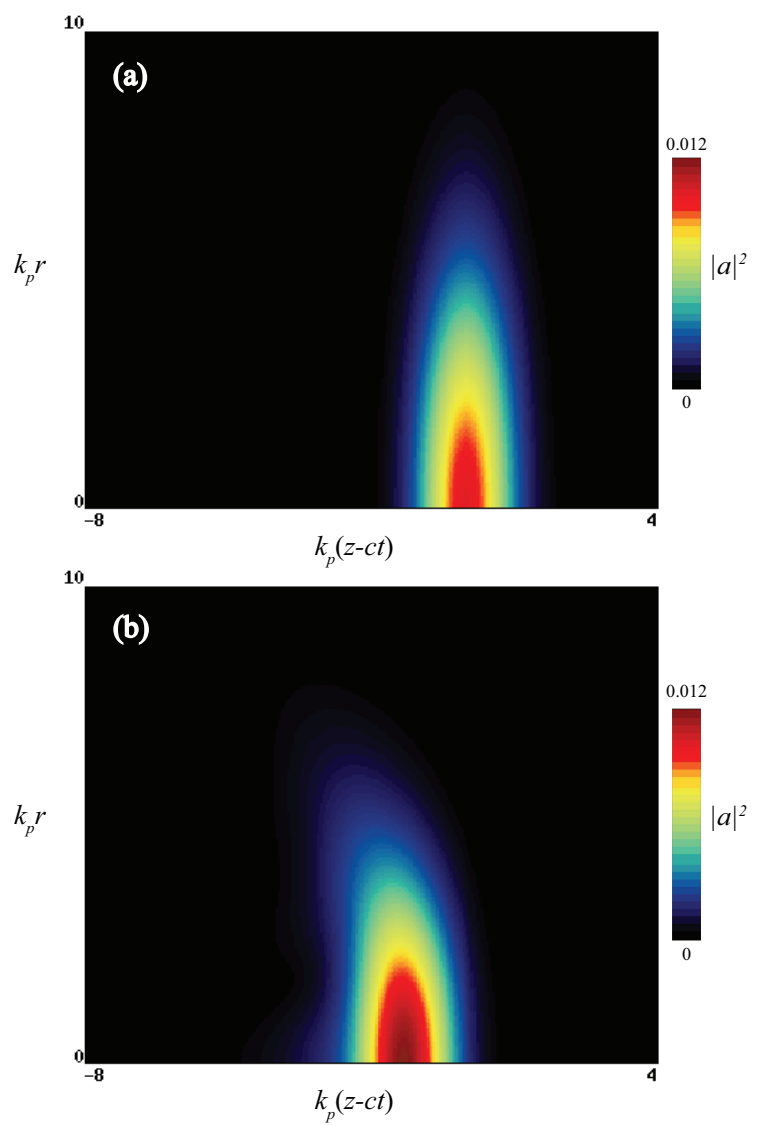

FIG. 5. Normalized laser intensity $\hat{a}^{2}$ at (a) $z=0$ and (b) $z=10 Z_{m}$, for the parameters $r_{i}=1.5 r_{m}$, $k_{0} / k_{p}=25, k_{p} L=1, a_{0}=0.01$, and $\lambda_{0}=0.8 \mu \mathrm{m}$.

a mismatched laser pulse with $r_{i}=1.3 r_{m}$, and (c) mismatched laser pulse with $r_{i}=1.5 r_{m}$. The laser and plasma parameters used in Fig. 4 are $k_{0} / k_{p}=25, k_{p} L=1, a_{0}=0.01$, and $\lambda_{0}=0.8 \mu \mathrm{m}$. Solid curves are numerical solutions of the full Maxwell equations coupled to the plasma response calculated using the code INF\&RNO ${ }^{24}$ and the dashed curves are calculated using the approximate solution Eq. (47). Note that the growth in the rms pulse length of the matched laser pulse [case (a) in Fig. 4] is due to dispersion via the laser bandwidth. Figure 4 shows good agreement between Eq. (47) and numerical solutions in the limit of a slightly mismatched laser propagating in a plasma channel.

The pulse lengthening is due to the slippage of the fundamental laser mode with respect to the higher-order laser modes in a plasma channel. This is illustrated in Fig. 5 where the normalized laser intensity $|\hat{a}|^{2}$ is shown for the parameters $k_{0} / k_{p}=25, k_{p} L=1, a_{0}=0.01$, and $\lambda_{0}=0.8 \mu \mathrm{m}$. Figure $5(\mathrm{a})$ is the initial laser profile, with initial mismatch $r_{i}=1.5 r_{m}$, and 
(b) is the laser profile after propagating $10 Z_{m}$. Figure $5(\mathrm{~b})$ shows the slippage of the laser centroid with respect to the initial position $\left(\beta_{G}<1\right)$. Also, one can observe the slippage of the $m=1$ mode with respect to the fundamental $m=0$ in a plasma channel, resulting in off-axis curvature and pulse lengthening.

\section{DISCUSSION}

In this work, a general expression for the laser group velocity was derived for a laser propagating in a plasma channel in the linear (low intensity, $a^{2} \ll 1$ ) limit. The group velocity was defined as the velocity of the normalized intensity-weighted centroid of the laser pulse. The general expression for the group velocity, Eq. (32), was obtained for an arbitrary Laguerre-Gaussian mode and is valid for mismatched propagation (an oscillating spot size) in a plasma channel. This expression for the group velocity reduces to the previously derived results for matched propagation (constant spot size) in a plasma channel or for a diffracting laser pulse (no plasma channel).

For a mismatched laser propagating in a plasma channel, in which the spot size undergoes strong oscillations, the group velocity is shown to be constant, independent of the propagation distance. The group velocity of a mismatched laser, for fixed plasma channel parameters, is shown to be slower than that of a matched laser. This reduction of the group velocity is due to the increase of the effective divergence angle of the mismatched laser within the channel. In particular, for the fundamental Gaussian mode, the effective transverse wavenumber $k_{\perp}$ that determines the group velocity $\beta_{g} \simeq 1-k_{p}^{2} / 2 k_{0}^{2}-k_{\perp}^{2} / 2 k_{0}^{2}$ is given by $k_{\perp}^{2} \simeq 4 / r_{m}^{2}$ for a matched laser. For a mismatched laser, $k_{\perp}^{2} \simeq\left(4 / r_{m}^{2}\right)\left(\left\langle r_{s}^{2}\right\rangle / r_{m}^{2}\right)$, where $\left\langle r_{s}^{2}\right\rangle^{1 / 2}$ is the rms value of the spot size in the plasma channel and $\left\langle r_{s}^{2}\right\rangle / r_{m}^{2}=\left(r_{ \pm}^{2} / r_{m}^{2}+r_{m}^{2} / r_{ \pm}^{2}\right) / 2>1$, with $r_{ \pm}$being the extremum of the spot size oscillation. For a higher-order mode laser, the additional transverse mode structure increases the effective transverse wavenumber, such that $k_{\perp}^{2} \rightarrow(1+2 m+p) k_{\perp}^{2}$, which decreases the laser group velocity.

The pulse length of a mismatch laser will lengthen as it propagates in a plasma channel. A mismatch laser can be decomposed into a sum of matched higher-order modes. Since the group velocity decreases with increasing mode number, the higher-order modes will slip behind the lower-order modes as the mismatched pulse propagates. This results in a distortion of the pulse profile, which becomes crescent-shaped as the higher-order modes 
begin to slip behind the fundamental mode (cf. Fig. 5). For a small mismatch, $\left(\delta r / r_{m}\right)^{2} \ll 1$, the rms laser pulse length $\sigma$ is given by $\left(\sigma / \sigma_{0}\right)^{2} \simeq 1+\left(z / Z_{S}\right)^{2}$, where $Z_{S}=\left(r_{m} / \delta r\right) k_{0} L Z_{m} / 4$. Pulse lengthening due to mode slippage will dominate over that due to laser bandwidth (dispersive spreading) provided $\left(\delta r / r_{m}\right)^{2} \gg\left(1+k_{p}^{2} r_{m}^{2} / 4\right)^{2} / k_{0}^{2} L^{2}$, i.e., for sufficiently long pulses.

The theory presented in this paper neglected nonlinear effects and is based on solutions to the linear wave equation. To check the validity of the analytical solutions in the limit of weakly-relativistic laser intensities, comparisons were made to numerical solutions using the nonlinear Maxwell-fluid code INF\&RNO. For example, for the parameters in Fig. 3(i) $\left(k_{p} r_{m}=\pi, k_{p} L=1\right)$, analytical and numerical results were in good agreement over many Rayleigh ranges for $a_{0}<0.5$, where corresponds to $P / P_{c}<0.1$. As $a_{0}$ increases, nonlinear laser evolution effects (self-focusing of the back of the pulse, laser depletion, and frequency red-shifting from plasma wave generation) causes the group velocity to decrease as a function of propagation distance. Minimization of these effects can be achieved by operating in a regime that minimizes the plasma wave excitation, i.e., $\left(P / P_{c}\right) k_{p}^{2} L^{2} \ll 1$.

One relevant application of this work is as a diagnostic of plasma channels using low intensity laser pulses. For example, measuring the delay induced by the plasma channel between two laser pulses of different frequencies can be used to determine the on-axis plasma density $n_{0}$ and the channel depth $\Delta n$ (i.e., the matched channel radius $r_{m}$, which is independent of laser frequency). An accurate determination of the plasma channel properties with this technique requires a detailed knowledge of the group velocity of a mismatched pulse, as described in this work. This diagnostic technique implicitly assumes longitudinal uniformity of the channel and, for nonuniform channels, provides a measure of the average channel density. In addition, this technique would require detailed knowledge of the lasers entering the channel, including size and mode content. Experimental implementation of this technique will be the subject of a future publication. ${ }^{21}$

\section{ACKNOWLEDGMENTS}

This work was supported by the Director, Office of Science, Office of High Energy Physics, of the U.S. Department of Energy under Contract No. DE-AC02-05CH11231. 


\section{Appendix A: Diffracting laser pulse}

In this Appendix we consider the general solution presented in Sec. II in the limit $\Delta n \rightarrow 0$ (or $r_{m} \rightarrow \infty$ ), with $r_{i}=r_{0}$, i.e., a diffracting laser pulse without a plasma channel. In this limit, the curvature, phase shift, and laser spot size are

$$
\begin{aligned}
\alpha_{0} & =\hat{z}, \\
\theta_{0} & =-(1+2 m+p) \tan ^{-1}(\hat{z}), \\
r_{s 0}^{2} & =r_{0}^{2}\left[1+\hat{z}^{2}\right],
\end{aligned}
$$

and Eq.(23) becomes

$$
\Im\left[\psi^{\prime}\right]=\frac{k_{0}^{-1} \hat{z}}{\left(1+\hat{z}^{2}\right)}\left[(1+2 m+p)-\frac{\left(1-\hat{z}^{2}\right)}{\left(1+\hat{z}^{2}\right)} \frac{r^{2}}{r_{0}^{2}}\right],
$$

where $\hat{z}=z / Z_{R}$. The laser centroid with respect to $\beta_{g 0}=1-k_{p}^{2} / 2 k_{0}^{2}$ [cf. Eq. (13) with $\left.r_{m} \rightarrow \infty\right]$ is $k_{0}\langle\zeta\rangle=-\hat{z}(1+2 m+p) / 2$, and the group velocity of a diffracting pulse is given by Eq. (35).

\section{Appendix B: Local group velocity}

Equation Eq. (30) describes the velocity of the global laser centroid Eq. (27). A local laser centroid $\zeta_{L}=\zeta_{L}(r, z)$ may be defined as

$$
\zeta_{L}=\frac{\int_{-\infty}^{\infty} d \zeta|\hat{a}|^{2} \zeta}{\int_{-\infty}^{\infty} d \zeta|\hat{a}|^{2}}
$$

Using Eq. (26), the local centroid of the laser intensity is $\zeta_{L}=-\Im\left[\psi^{\prime}\right]$, where the imaginary component of the non-paraxial exponent is given by Eq. (23). The velocity associated with the rate of change of the local laser centroid is $\beta_{L}=\beta_{g 0}+d \zeta_{L} / d z$, and is, in general, a function of the laser propagation distance and the radial coordinate $\beta_{L}(r, z)$. Evaluating the local laser velocity using Eq. (23) yields 


$$
\begin{aligned}
& \beta_{L} \simeq 1-\frac{k_{p}^{2}}{2 k_{0}^{2}}-\frac{2(1+2 m+p)}{k_{0}^{2} r_{m}^{2}}\left\{\frac{r_{i}^{2} r_{m}^{2} \sec ^{2}\left(z / Z_{m}\right)}{r_{i}^{4}+r_{m}^{4} \tan ^{2}\left(z / Z_{m}\right)}\right. \\
& \left.-\frac{2 r_{i}^{2} r_{m}^{2}\left(r_{m}^{4}-r_{i}^{4}\right)\left(z / Z_{m}\right) \tan \left(z / Z_{m}\right) \sec ^{2}\left(z / Z_{m}\right)}{\left[r_{i}^{4}+r_{m}^{4} \tan ^{2}\left(z / Z_{m}\right)\right]^{2}}\right\} \\
& +\frac{2}{k_{0}^{2} r_{m}^{2}}\left\{\frac { ( r _ { m } ^ { 4 } - r _ { i } ^ { 4 } ) ( r _ { i } ^ { 8 } - r _ { m } ^ { 8 } ) } { [ r _ { m } ^ { 4 } + r _ { i } ^ { 4 } - ( r _ { m } ^ { 4 } - r _ { i } ^ { 4 } ) \operatorname { c o s } ( 2 z / Z _ { m } ) ] ^ { 3 } } \left[3+2\left(z / Z_{m}\right) \sin \left(4 z / Z_{m}\right)+\cos \left(4 z / Z_{m}\right)\right.\right. \\
& \left.\left.+4 \frac{\left(r_{i}^{8}-6 r_{i}^{4} r_{m}^{4}+r_{m}^{8}\right)}{\left(r_{i}^{8}-r_{m}^{8}\right)}\left(z / Z_{m}\right) \sin \left(2 z / Z_{m}\right)+4 \frac{\left(r_{i}^{8}+r_{m}^{8}\right)}{\left(r_{i}^{8}-r_{m}^{8}\right)} \cos \left(2 z / Z_{m}\right)\right]\right\} \frac{r^{2}}{r_{m}^{2}} .
\end{aligned}
$$

Note that the local group velocity Eq. (B2) is based on a perturbation expansion and is valid only for phase shifts such that $k_{0} \psi^{\prime} \ll k_{0} / \delta k \sim k_{0} L$. This validity condition may be violated for sufficiently long propagation distances or at large radii.

For matched laser propagation $\left(r_{i}=r_{m}\right), d \zeta_{L} / d z=0$ and the local laser velocity is equal to the global laser group velocity $\beta_{L}=\beta_{G}=\beta_{g 0}$. In the limit of a diffracting laser pulse without a plasma channel (cf. Appendix A), the local laser velocity Eq. (B2) reduces to

$$
\beta_{L} \simeq 1-\frac{k_{p}^{2}}{2 k_{0}^{2}}-\frac{2}{k_{0}^{2} r_{0}^{2}}\left[\frac{(1+2 m+p)\left(1-\hat{z}^{2}\right)}{\left(1+\hat{z}^{2}\right)^{2}}+\frac{\left(1-6 \hat{z}^{2}+\hat{z}^{4}\right)}{\left(1+\hat{z}^{2}\right)^{3}} \frac{r^{2}}{r_{0}^{2}}\right]
$$

At the focus, $\hat{z}=0$, and on axis, $r=0$, Eq. (B3) reduces to $\beta_{L} \simeq 1-k_{p}^{2} / 2 k_{0}^{2}-2(1+2 m+$ p) $/ k_{0}^{2} r_{0}^{2}$. Asymptotically, $\hat{z} \rightarrow \infty$, Eq. (B3) has the limit $\beta_{L} \simeq 1-k_{p}^{2} / 2 k_{0}^{2}$.

\section{REFERENCES}

${ }^{1}$ E. Esarey, P. Sprangle, J. Krall, and A. Ting, IEEE J. Quantum Electron. 33, 1879 (1997).

${ }^{2}$ C. G. Durfee III and H. M. Milchberg, Phys. Rev. Lett. 71, 2409 (1993).

${ }^{3}$ E. Esarey, C. B. Schroeder, and W. P. Leemans, Rev. Mod. Phys. 81, 1229 (2009).

${ }^{4}$ J. P. Palastro, T. M. Antonsen, S. Morshed, A. G. York, and H. M. Milchberg, Phys. Rev. E 77, 036405 (2008).

${ }^{5}$ A. G. York, H. M. Milchberg, J. P. Palastro, and T. M. Antonsen, Phys. Rev. Lett. 100, 195001 (2008).

${ }^{6}$ H. M. Milchberg, C. G. Durfee III, and T. J. McIlrath, Phys. Rev. Lett. 75, 2494 (1995).

${ }^{7}$ D. M. Gaudiosi, B. Reagan, T. Popmintchev, M. Grisham, M. Berrill, O. Cohen, B. C. Walker, M. M. Murnane, H. C. Kapteyn, and J. J. Rocca, Phys. Rev. Lett. 96, 203001 (2006). 
${ }^{8}$ H. M. Milchberg, T. R. Clark, C. G. Durfee III, T. M. Antonsen, Jr., and P. Mora, Phys. Plasmas 3, 2149 (1996).

${ }^{9}$ D. V. Korobkin, C. H. Nam, S. Suckewer, and A. Goltsov, Phys. Rev. Lett. 77, 5206 (1996).

${ }^{10}$ K. A. Janulewicz, J. J. Rocca, F. Bortolotto, M. P. Kalachnikov, V. N. Shlyaptsev, W. Sandner, and P. V. Nickles, Phys. Rev. A 63, 033803 (2001).

${ }^{11}$ C. G. Durfee III, J. Lynch, and H. M. Milchberg, Phys. Rev. E 51, 2368 (1995).

${ }^{12}$ Y. Ehrlich, C. Cohen, A. Zigler, J. Krall, P. Sprangle, and E. Esarey, Phys. Rev. Lett. 77, 4186 (1996).

${ }^{13}$ P. Volfbeyn, E. Esarey, and W. Leemans, Phys. Plasmas 6, 2269 (1999).

${ }^{14}$ T. Hosokai, M. Kando, H. Dewa, H. Kotaki, S. Kondo, N. Hasegawa, K. Nakajima, and K. Horioka, Opt. Lett. 25, 10 (2000).

${ }^{15}$ T. R. Clark and H. M. Milchberg, Phys. Rev. E 61, 1954 (2000).

${ }^{16}$ A. Butler, D. J. Spence, and S. M. Hooker, Phys. Rev. Lett. 89, 185003 (2002).

${ }^{17}$ B. M. Luther, Y. Wang, M. C. Marconi, J. L. A. Chilla, M. A. Larotonda, and J. J. Rocca, Phys. Rev. Lett. 92, 235002 (2004).

${ }^{18}$ C. G. R. Geddes, C. Toth, J. van Tilborg, E. Esarey, C. B. Schroeder, J. Cary, and W. P. Leemans, Phys. Rev. Lett. 95, 145002 (2005).

${ }^{19}$ A. J. Gonsalves, K. Nakamura, C. Lin, J. Osterhoff, S. Shiraishi, C. B. Schroeder, C. G. R. Geddes, C. Tóth, E. Esarey, and W. P. Leemans, Phys. Plasmas 17, 056706 (2010).

${ }^{20}$ E. Esarey and W. P. Leemans, Phys. Rev. E 59, 1082 (1999).

${ }^{21}$ J. van Tilborg, et al., (2011), submitted for publication.

${ }^{22}$ C. D. Decker and W. B. Mori, Phys. Rev. Lett. 72, 490 (1994).

${ }^{23}$ C. B. Schroeder, C. Benedetti, E. Esarey, and W. P. Leemans, Phys. Rev. Lett. 106, 135002 (2011).

${ }^{24}$ C. Benedetti, C. B. Schroeder, E. Esarey, C. G. R. Geddes, and W. P. Leemans, in Proc. of 2010 Advanced Accelerator Concepts Workshop, Vol. 1299, edited by G. Nusinovich and S. Gold (AIP, NY, 2010) pp. 250-255; C. Benedetti, W. P. Leemans, E. Esarey, and C. B. Schroeder, in Proc. of PAC11 (JACoW, 2011) p. MOP082.

${ }^{25}$ P. Mora and T. M. Antonsen, Jr., Phys. Plasmas 4, 217 (1997). 